\title{
Correlation between grain growth and disk geometry in Herbig Ae/Be systems
}

\author{
B. Acke ${ }^{1}$, M. E. van den Ancker $^{2}$, C. P. Dullemond ${ }^{3}$, R. van Boekel ${ }^{4}$, and L. B. F. M. Waters ${ }^{1,4}$ \\ ${ }^{1}$ Instituut voor Sterrenkunde, Katholieke Universiteit Leuven, Celestijnenlaan 200B, 3001 Leuven, Belgium \\ e-mail: bram.acke@ster. kuleuven.ac.be \\ 2 European Southern Observatory, Karl-Schwarzschild Strasse 2, 85748 Garching bei München, Germany \\ 3 Max-Planck-Institut für Astrophysik, Karl-Schwarzschildstrasse 1, Postfach 1317, 85748 Garching bei München, Germany \\ 4 Astronomical Institute “Anton Pannekoek”, University of Amsterdam, Kruislaan 403, 1098 SJ Amsterdam, The Netherlands
}

Received 4 February 2004 / Accepted 24 April 2004

\begin{abstract}
We have calculated the (sub-)mm spectral indices of $26 \mathrm{Herbig} \mathrm{Ae/Be} \mathrm{stars,} \mathrm{for} \mathrm{which} \mathrm{we} \mathrm{can} \mathrm{determine} \mathrm{the} \mathrm{infrared}$ spectral energy distribution (SED). We find a clear correlation between the strength of the ratio of the near- to mid-infrared excess of these sources, and the slope of the (sub-)mm energy distribution. Based on earlier multi-dimensional modeling of disks around Herbig Ae stars, we interpret this as a correlation between the geometry of the disk (flared or self-shadowed) and the size of the grains: self-shadowed disks have, on average, larger grains than their flared counterparts. These data suggest that the geometry of a young stellar disk evolves from flared to self-shadowed.
\end{abstract}

Key words. stars: circumstellar matter - stars: planetary systems: protoplanetary disks - stars: pre-main-sequence

\section{Introduction}

Herbig Ae/Be (HAEBE) stars are the somewhat more massive analogues of the $\mathrm{T}$ Tauri stars, which are low-mass young-stellar objects. The spectral energy distribution (SED) of HAEBE stars is characterized by the presence of an infrared (IR) flux excess, due to circumstellar dust and gas. The geometry of this circumstellar matter is believed to be disk-like (e.g., Mannings \& Sargent 1997, 2000; Fuente et al. 2003).

Meeus et al. (2001, henceforth M01) classified 14 isolated HAEBE sample stars into two groups, based on the shape of the SED. Group I contains the sources in which a rising midIR $(20-100 \mu \mathrm{m})$ flux excess is observed; these sources have an SED that can be fitted with a power-law and a black-body continuum. Group II sources have more modest mid-IR excesses; their IR SEDs can be reconstructed by a power-law only. M01 suggested phenomologically that the difference between the two groups is related to the disk geometry.

Dullemond (2002) (henceforth D02) and Dullemond \& Dominik (2004) (henceforth D04) have modelled young stellar disks with a self-consistent model based on 2-D radiative transfer coupled to the equation of vertical hydrostatics. The model consists of a disk with an inner hole $(\sim 0.5 \mathrm{AU})$, a puffed-up inner rim and an outer part. The outer part of the disk can be flared (as in Chiang \& Goldreich 1997), but can also lie entirely in the shade of the inner rim. The SEDs of flared disks display a strong mid-IR flux excess, while self-shadowed disks have a much steeper slope towards long wavelengths. D02 can explain the difference in SED shape in HAEBEs (as expressed by the classification of M01) as the result of a different disk geometry; flared-disks systems have group I SEDs, sources with self-shadowed disks can be linked to group II objects.

In this paper we calculate the spectral index of the (sub-)mm (350-2700 $\mu \mathrm{m})$ SED for a sample of 26 HAEBE stars. We look for a connection between this index and the geometry of the circumstellar disk. In Sect. 2 we classify the sample sources and present the computed (sub-)mm spectral indices. Interpretations of the results obtained in the latter section are given in Sect. 3. We summarize our conclusions in the final Sect. 4.

\section{Observations and analysis}

The sources were selected based on Tables 1 and 2 in the latest catalog of HAEBE stars (Thé et al. 1994). We enlarged our list of HAEBEs with objects from the sample studied by Malfait et al. (1998) that satisfy the criteria postulated by Thé et al. We excluded sources that have the amorphous 10 micron silicate feature in absorption (Acke \& van den Ancker 2004, henceforth AV04), since these sources are probably in an earlier evolutionary stage and thus highly embedded. Early-B stars are excluded as well, because the disk-like nature of the circumstellar matter in these sources is still a subject of debate (e.g. see review by Natta et al. 2000, on HAEBE disks in the book Protostars and Planets $I V$ ). For the remaining set of stars, we gathered broad-band $J, H, K, L$ and $M$ photometry and IRAS 12, 25 and 

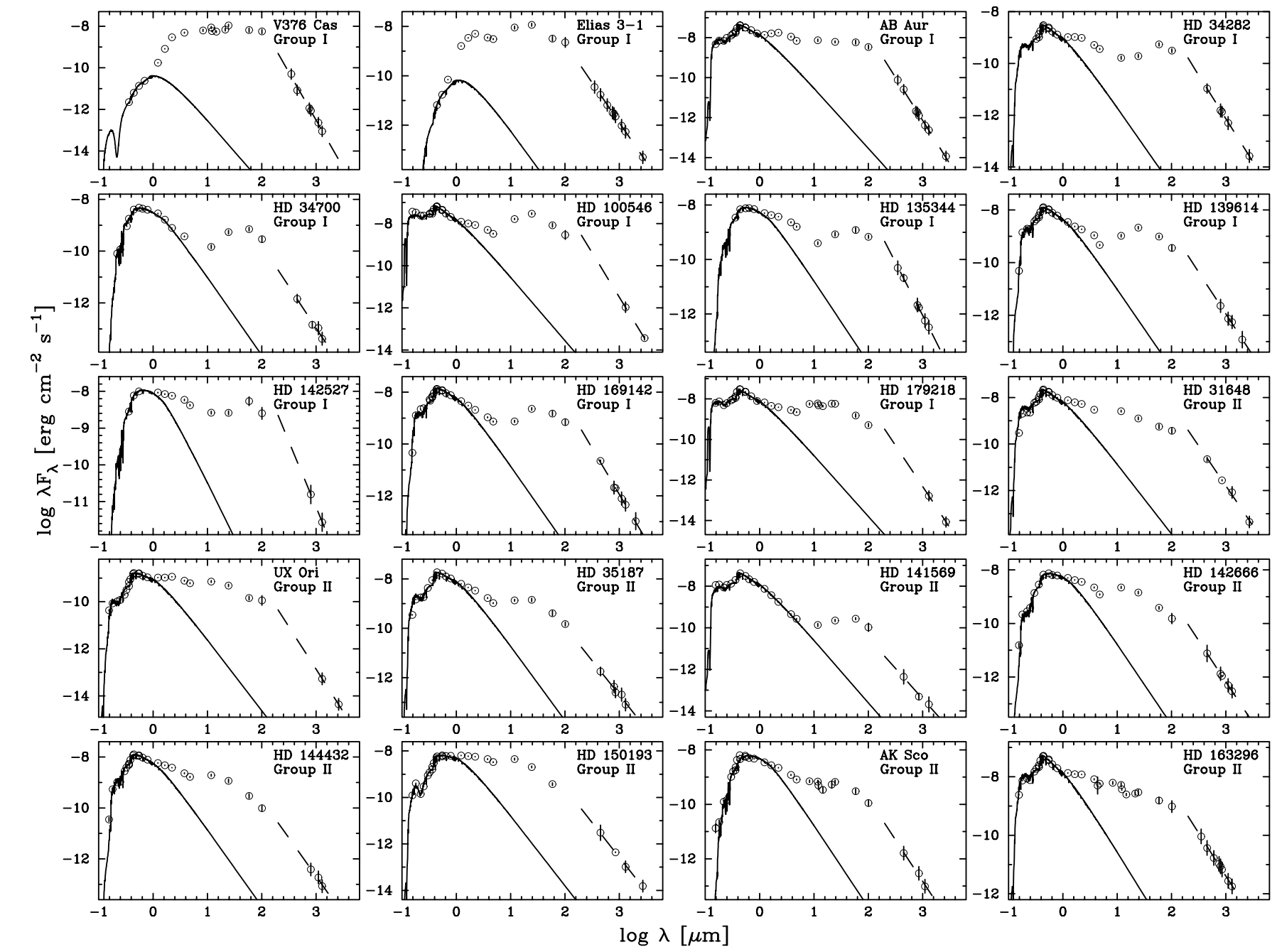

Fig. 1. The SEDs of the sample sources with sufficient data to determine $n$. The circles indicate photometric measurements with error bars. The full line represents a reddened Kurucz model corresponding to the photosphere of the central star. The model was fitted to the optical photometric data. The (sub-)mm slope of the SED is given by the dashed line.

60 micron measurements in the literature. For 26 of the stars for which this IR photometry was available, also (sub-)mm observations were present in the literature. Our final sample consists of these sources.

At (sub-)mm wavelengths, one observes the Rayleigh-Jeans part of the SED of the cold grains. Grains that are large compared to the wavelength at which they radiate, have a spectrum $\lambda \mathcal{F}_{\lambda} \propto \lambda^{-n}$ with spectral index $n=3$ (black body). When the grains are smaller, $n$ is larger, if the dust is optically thin at these wavelengths. Hence the (sub-)mm spectral index can be interpreted in terms of the grains size distribution of the cold dust in the circumstellar disk (Sylvester et al. 1996; van den Ancker et al. 2000; M01). Nevertheless, this should be done with some caution, as we will discuss in the next section. We compute the (sub-)mm spectral index fitting a power law to the photometry between 350 and $2700 \mu \mathrm{m}$. In case only one (sub-)mm photometric point was available, we determined a lower limit to the (sub-)mm spectral index using the (sub-)mm data point and the IRAS 100 micron measurement. For BF Ori, we used the IRAS 60 micron measurement, since the IRAS 100 micron measurement resulted in an upper limit in this case.

We note that the fluxes used to compute $n$ come from a variety of literature sources, both single-dish and interferometric. However, the vast majority of measurements come from single-dish instruments. There are only four sample sources (V376 Cas, Elias 3-1, HD 34282 and UX Ori) in which interferometric data are used. The latter two sources (HD 34282 and UX Ori) appear rather isolated (Malfait et al. 1998). Therefore the interferometric fluxes are expected to be identical to those obtained with single-dish measurements. For the two sources with possible envelope emission (V376 Cas and Elias 3-1: Henning et al. 1998; di Francesco et al. 1997) for which we have mixed interferometric and single-dish measurements, we do not note any systematic differences between the two. In Fig. 1 the SEDs of the sample sources with sufficient (sub-)mm photometry to determine $n$ are presented.

The IR SED of the sample sources can be characterized with two quantities: the ratio of the near-IR luminosity $L_{\mathrm{NIR}}$ (derived from the broad-band $J, H, K, L$ and 


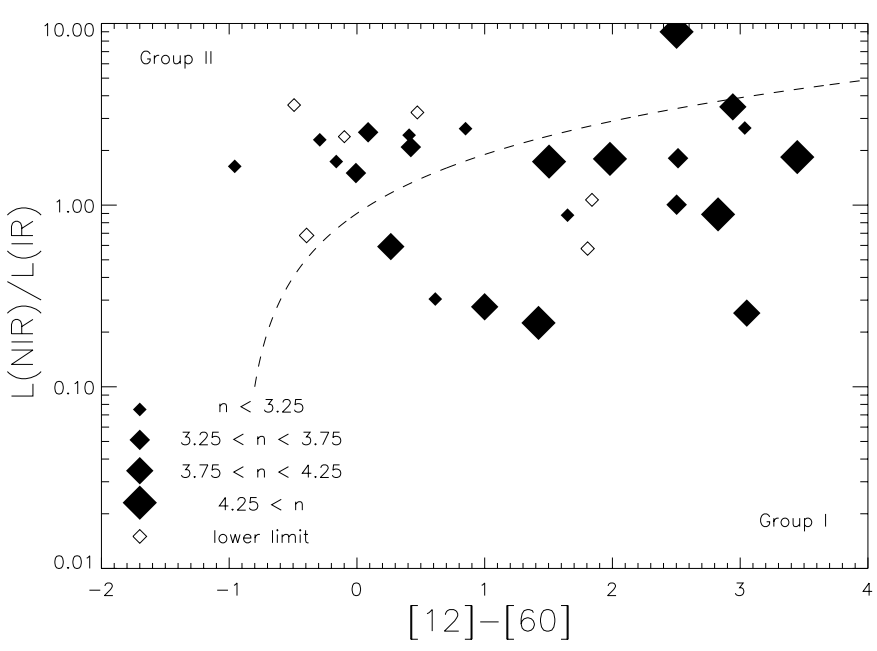

Fig. 2. Diagram based on van Boekel et al. (2003). The ratio $L_{\mathrm{NIR}} / L_{\mathrm{IR}}$ is plotted versus the non color-corrected IRAS [12]-[60] color. The dashed line empirically separates the group II (left) and the group I (right) sources. The size of the plot symbol is proportional to the (sub-)mm spectral index $n$. Open plot symbols indicate lower limits.

$M$ photometry) and the mid-IR luminosity $L_{\mathrm{IR}}$ (the corresponding quantity based on IRAS 12, 25 and 60 micron measurements), and the non-color corrected IRAS [12]-[60] color. Both parameters compare the near-IR photometric data to the mid-IR IRAS measurements (van Boekel et al. 2003; Dullemond et al. 2003). The near-IR SED is expected to be similar for all HAEBEs (Natta et al. 2001), while the major differences occur in the mid-IR SED. The luminosity ratio $L_{\mathrm{NIR}} / L_{\mathrm{IR}}$ represents the strength of the near-IR compared to the mid-IR excess, which is lower for group I than group II sources. The shape of the mid-IR SED of a group I source is "doublepeaked" compared to the SED of a group II member. The IRAS [12]-[60] color index provides a quantitative measure for this difference in SED shape. Group I sources are redder than their group II counterparts.

Following van Boekel et al. (2003), we use these quantities to distinguish between group I and II in the classification of M01. We plotted all sample stars in Fig. 2. The dashed line in the diagram represents $L_{\mathrm{NIR}} / L_{\mathrm{IR}}=([12]-[60])+0.9$, which empirically provides the best separation between the two groups. The sources defined by M01 as group I and group II do indeed occupy the correct location in this diagram using this separating line. The diagram provides a quantitative alternative to the subjective classification method of M01. In Fig. 2, the sizes of the plotting symbols are proportional to the (sub-)mm spectral index.

The computed (sub-)mm spectral indices $n$ of all sample stars are listed in Table ??. The error on the spectral index $\sigma_{n}$ reflects the dispersion on the fitted line. Note that the values of $n$ show significant variations from source to source. The (sub-)mm spectral indices range from the default ISM value of optically thin dust $n \approx 5$ (Miyake \& Nakagawa 1993) to the optically thin black-body value $n=3$. The average values for group I and group II are also indicated in Table ??. The error on the average is given by $\left(\sum_{j} 1 / \sigma_{n, j}\right)^{-1}$ in which the $\sigma_{n, j}$ represent the errors on the individual $n$-values in the group.

\section{Discussion and interpretations}

\subsection{Comparison with other articles}

Hillenbrand et al. (1992, henceforth H92) also classified HAEBE stars in groups. We stress that this was performed based on the near-IR $(\sim 3.5-10.6 \mu \mathrm{m})$ spectral index. Their classification is not linked to the M01 classification used in this paper; both H92 group I and II contain M01 group I as well as group II sources. H92 group III is not relevant for sources with significant dust disks. The classification of M01 focusses on the $\sim 20-100 \mu \mathrm{m}$ SED, which makes it well-suited for this observational analysis.

There exists a large variety of models for the SEDs of HAEBEs in the literature (e.g. H92 Natta et al. 1992, 1993; Mannings \& Emerson 1994). While most of these (disk and/or envelope) models give satisfactory fits to the mid-IR SED, they have difficulty to accurately describe the near-IR bump that is typical for HAEBEs. One of the main improvements in the model of Dullemond et al. (2001) and DD04 is the inclusion of the puffed-up inner rim, which generates this near-IR bump, as was first suggested by Natta et al. (2001) and Tuthill et al. (2001). Furthermore, the Dullemond et al. model explains in a natural way the roughly two types of IR SEDs (M01 group I and II) observed in HAEBE stars. The quantitative fine-tuned modelling of the SEDs of our sample stars, however, is beyond the scope of the present observational article.

\subsection{Grain growth in circumstellar disks around HAEBEs}

If the disks surrounding HAEBE stars are optically thin at (sub-)mm wavelengths, the spectral index of the SED in this wavelength range will be independent of disk geometry. In that case, intrinsic properties of the cold grains in the outer parts of the disk must be responsible for the observed variations in the (sub-)mm spectral index.

In order to test the hypothesis that the circumstellar disks in our sample are indeed optically thin at (sub-)mm wavelengths, we have computed the radii of the circumstellar disks of our sample sources based on the measured $1.3 \mathrm{~mm}$ flux, assuming the simple model of an optically thick disk with a black-body temperature of $40 \mathrm{~K}$. The resulting radii are of the order of 10-20 AU. Comparing this to the observed radii of Herbig Ae stars (e.g., Mannings \& Sargent 1997; Natta et al. 2004; Testi et al. 2003; Piétu et al. 2003) or the typical disk sizes of T Tauri stars $(\sim 100 \mathrm{AU})$, the estimated radii of our sample-star disks seem too small. It is therefore unlikely that the disks of our sample stars are optically thick at (sub-)mm wavelengths.

Supposing an optically thin disk at (sub-)mm wavelengths and a typical grain temperature of $40 \mathrm{~K}$, one can estimate the disk mass from the $1.3 \mathrm{~mm}$ flux. Using formula (14) in Hillenbrand et al. (1992), we derived disk masses for the sample sources between $5.6 \times 10^{-4}$ and $1.5 \times 10^{-1} M_{\odot}$. This compares well to the maximum disk mass of $0.3 M_{\star}$, set by gravitational instabilities in the disk (e.g., Gammie 2001). When the disk is optically thick, its mass would be several factors higher, 
Table 1. The (sub-)mm photometry and spectral index of our sample sources. The sources are sorted by group. The photometry gathered from the literature is listed in Cols. (3) to (10). The unit is Jy. Between brackets, the observing wavelength in micron is given. In Col. (11), the disk mass estimated based on the 1300 micron flux is presented. The (sub-)mm spectral index $n$ and error $\sigma_{n}$ are given in Cols. (12) and (13) respectively. The references are indicated in Col. (14). The mean spectral index of each group is presented as well. ${ }^{\star}$ These stars are UX Orionis objects according to the definition of Dullemond et al. (2003). Note that the mean spectral index of these objects (3.13 \pm 0.03 ) is in good agreement with their believed group II nature (Dullemond et al. 2003). ${ }^{b}$ Measurement at $600 \mu \mathrm{m}: 1.3 \pm 0.1 \mathrm{Jy}$ for Elias 3-1 and $3.38 \pm 0.09 \mathrm{Jy}$ for HD $163296 .{ }^{\sharp}$ : average of measurements by Ref. 15 and $17 .{ }^{\dagger}$ Based on the 1100 micron flux. ${ }^{a}$ Measurement at $2 \mathrm{~mm} ;{ }^{b}$ measurement at $2.6 \mathrm{~mm} ;{ }^{c}$ measurement at $2.9 \mathrm{~mm}$.

\begin{tabular}{|c|c|c|c|c|c|c|c|c|c|c|c|c|c|}
\hline \multicolumn{14}{|c|}{ (sub-)mm photometry and spectral index } \\
\hline Object & Group & [350] & [450] & [750] & [800] & {$[850]$} & {$[1100]$} & {$[1300]$} & [2700] & $M_{\mathrm{disk}}\left[M_{\odot}\right]$ & $n$ & $\sigma_{n}$ & Ref. \\
\hline (1) & (2) & (3) & (4) & $(5)$ & (6) & (7) & (8) & (9) & $(10)$ & (11) & (12) & (13) & (14) \\
\hline V376 Cas & $\mathrm{I}$ & $5.8 \pm 0.5$ & $1.3 \pm 0.2$ & $0.28 \pm 0.04$ & $0.23 \pm 0.02$ & & $0.08 \pm 0.02$ & $0.038 \pm 0.006$ & $<0.0048$ & $8.8 \times 10^{-2}$ & 4.59 & 0.21 & $3,7,13$ \\
\hline Elias 3-1 ${ }^{b}$ & I & $4.1 \pm 0.6$ & $2.6 \pm 0.4$ & $0.8 \pm 0.1$ & $0.78 \pm 0.05$ & $0.64 \pm 0.05$ & $0.35 \pm 0.05$ & $0.23 \pm 0.02$ & $0.05 \pm 0.01$ & $3.5 \times 10^{-2}$ & 3.22 & 0.03 & $1,3,7$ \\
\hline AB Aur & I & $9 \pm 1$ & $3.8 \pm 0.6$ & $0.53 \pm 0.08$ & $0.50 \pm 0.05$ & $0.36 \pm 0.07$ & $0.15 \pm 0.02$ & $0.10 \pm 0.02$ & $0.011 \pm 0.005$ & $1.2 \times 10^{-2}$ & 4.32 & 0.09 & $3,7,8$ \\
\hline HD $34282^{\star}$ & I & & $1.6 \pm 0.2^{\sharp}$ & & $0.41 \pm 0.03$ & $0.38 \pm 0.02$ & $0.18 \pm 0.02$ & $0.11 \pm 0.01$ & $0.024 \pm 0.003^{b}$ & $1.0 \times 10^{-1}$ & 3.36 & 0.04 & $9,14,15,17$ \\
\hline HD 34700 & I & & $0.22 \pm 0.04$ & & & $0.041 \pm 0.002$ & $0.04 \pm 0.01$ & $0.018 \pm 0.002$ & & $1.2 \times 10^{-2}$ & 3.64 & 0.34 & $15,17,19$ \\
\hline HD 97048 & I & & & & & & & $0.45 \pm 0.03$ & & $9.2 \times 10^{-2}$ & $>2.94$ & & 4 \\
\hline HD 100453 & I & & & & & & & $0.27 \pm 0.02$ & & $2.1 \times 10^{-2}$ & $>2.82$ & & 10 \\
\hline HD 100546 & I & & & & & & & $0.47 \pm 0.02$ & $0.036 \pm 0.004^{c}$ & $2.9 \times 10^{-2}$ & 4.20 & 0.50 & 5,18 \\
\hline HD 135344 & I & $6 \pm 2$ & $3.2 \pm 0.2$ & & $0.57 \pm 0.02$ & $0.49 \pm 0.01$ & $0.21 \pm 0.01$ & $0.14 \pm 0.02$ & $<0.076^{a}$ & $1.6 \times 10^{-2}$ & 3.89 & 0.05 & $2,15,16$ \\
\hline HD 139614 & I & & & & $0.61 \pm 0.03$ & & $0.27 \pm 0.01$ & $0.24 \pm 0.01$ & $0.08 \pm 0.01^{a}$ & $2.7 \times 10^{-2}$ & 3.17 & 0.19 & 15 \\
\hline HD 142527 & I & & & & $4.2 \pm 0.5$ & & & $1.19 \pm 0.03$ & $0.047 \pm 0.006^{c}$ & $1.5 \times 10^{-1}$ & 3.58 & 0.41 & 18,19 \\
\hline HD 169142 & I & & $3.3 \pm 0.1$ & & $0.55 \pm 0.03$ & $0.57 \pm 0.01$ & $0.29 \pm 0.01$ & $0.20 \pm 0.02$ & $0.07 \pm 0.02^{a}$ & $2.4 \times 10^{-2}$ & 3.56 & 0.11 & 15,16 \\
\hline HD 179218 & I & & & & & & & $0.071 \pm 0.007$ & $0.008 \pm 0.001$ & $2.4 \times 10^{-2}$ & 4.06 & 0.26 & 9 \\
\hline Mean & I & & & & & & & & & & $\mathbf{3 . 6 0}$ & 0.09 & \\
\hline HD 31648 & II & & $3.3 \pm 0.3$ & & & $0.78 \pm 0.02$ & & $0.36 \pm 0.02$ & $0.04 \pm 0.01$ & $3.6 \times 10^{-2}$ & 3.44 & 0.18 & 8,16 \\
\hline UX Ori $\star$ & II & & & & & & & $0.020 \pm 0.002$ & $0.0038 \pm 0.0004^{b}$ & $1.6 \times 10^{-2}$ & 3.20 & 0.29 & 12 \\
\hline HD 35187 & II & & $0.27 \pm 0.05$ & & $0.12 \pm 0.02$ & $0.06 \pm 0.005$ & $0.08 \pm 0.01$ & $0.035 \pm 0.002$ & & $5.0 \times 10^{-3}$ & 2.77 & 0.38 & $15,17,19$ \\
\hline BF Ori ${ }^{\star}$ & II & & & & & & & $0.006 \pm 0.002$ & & $1.0 \times 10^{-2}$ & $>2.82$ & & 11 \\
\hline HD 104237 & II & & & & & & & $0.09 \pm 0.02$ & & $7.8 \times 10^{-3}$ & $>2.79$ & & 5 \\
\hline HD 141569 & II & & $0.07 \pm 0.02$ & & & $0.014 \pm 0.002$ & $<0.036$ & $0.009 \pm 0.004$ & & $5.6 \times 10^{-4}$ & 2.92 & 0.38 & $15,17,19$ \\
\hline HD $142666^{\star}$ & II & & $1.14 \pm 0.04$ & & $0.35 \pm 0.02$ & $0.313 \pm 0.005$ & $0.18 \pm 0.01$ & $0.127 \pm 0.09$ & $<0.063^{a}$ & $1.6 \times 10^{-2}$ & 3.07 & 0.01 & 15,16 \\
\hline HD 144432 & II & & & & $0.10 \pm 0.03$ & & $0.07 \pm 0.01$ & $0.037 \pm 0.003$ & & $4.9 \times 10^{-3}$ & 3.00 & 0.62 & 15,19 \\
\hline HD 150193 & II & & $0.5 \pm 0.2$ & & & $0.12 \pm 0.02$ & & $0.05 \pm 0.01$ & $0.014 \pm 0.003$ & $6.3 \times 10^{-3}$ & 2.97 & 0.11 & 6,8 \\
\hline AK Sco & II & & $0.24 \pm 0.03$ & & & $0.083 \pm 0.009$ & $0.036 \pm 0.004$ & & & $3.1 \times 10^{-3 \dagger}$ & 3.05 & 0.35 & 6 \\
\hline HD $163296^{b}$ & II & $10.7 \pm 0.7$ & $5.6 \pm 0.4$ & $2.65 \pm 0.08$ & $2.32 \pm 0.04$ & $1.92 \pm 0.03$ & $1.02 \pm 0.05$ & $0.78 \pm 0.03$ & & $6.8 \times 10^{-2}$ & 2.93 & 0.09 & 7 \\
\hline WW Vul` & II & & & & & & & $0.011 \pm 0.001$ & & $1.3 \times 10^{-2}$ & $>2.61$ & & 11 \\
\hline SV Cep ${ }^{\star}$ & II & & & & & & & $0.007 \pm 0.002$ & & $8.6 \times 10^{-3}$ & $>3.18$ & & 11 \\
\hline Mean & $\overline{\text { II }}$ & & & & & & & & & & 3.06 & 0.06 & \\
\hline
\end{tabular}

References:

1 Beckwith \& Sargent (1991)

2 Coulson \& Walther (1995).

3 di Francesco et al. (1997).

Henning et al. (1993).

Henning et al. (1994).

Jensen et al. (1996).

7 Mannings (1994).
8 Mannings \& Sargent (1997)

9 Mannings \& Sargent (2000)

10 Meeus et al. (2003).

11 Natta et al. (1997).

12 Natta et al. (1999).

13 Osterloh \& Beckwith (1995)

14 Piétu et al. (2003).
15 Sylvester et al. (1996).

16 Sandell \& Weintraub, AAS Poster (2003).

17 Sheret et al. (2004).

18 van den Ancker (unpublished ATCA data.)

19 Walker \& Butner (1995). 
which in some cases (e.g. HD 34282) would be close to this theoretical limit.

We have determined the value of the slope of the (sub-)mm SED by means of a power law fitted to the data between 350 and $2700 \mu \mathrm{m}$. It has not been proven that Herbig Ae/Be disks are still optically thin at the shortest wavelengths of this interval. However, for the sources for which we have measurements at the shorter wavelengths, we can also compute the (sub-)mm slope based on the longer-wavelength data only. There is no significant difference between the two. Hence, the slope of the energy distribution down to 350 and 450 micron does not deviate significantly from a power law. This shows that the optical depth at these wavelengths is not very different from that at $1.3 \mathrm{~mm}$. Another way of saying this is that our results would not change when omitting the 350 and 450 micron data.

Laboratory studies of the emission properties of dust species with astronomical relevance (e.g., Mennella et al. 1998; Colangeli et al. 2001) show that variations in chemical composition only exert a very mild influence on the (sub-)mm emission characteristics of dust particles. Particle size, on the other hand, has quite a large influence on the (sub-)mm emission. Therefore, and in accordance with previous authors (e.g., Mannings \& Emerson 1994; van den Ancker et al. 2000; Bouwman et al. 2000; Testi et al. 2003), we attribute the differences in (sub-)mm -spectral-energy-distribution slope to variations in dust particle size.

It is clear from Fig. 2 and Table ?? that the (sub-)mm spectral indices and the classification of the sample stars are correlated. Group I sources have an average spectral index $n$ equal to $3.60 \pm 0.09$. In group II, $n$ is $3.06 \pm 0.06$. Figure 3 describes this correlation alternatively. The spectral index is plotted versus the flux excess at 60 micron. The latter quantity is the difference between the measured flux and the flux of the reddened Kurucz model (see Fig. 1) at this wavelength. Group I objects, which are by definition brighter mid-IR sources, indeed display higher values for $n$. We interpret this correlation in terms of a correlation between the grain size distribution of the cold grains and the disk geometry. Group I sources (flared-disk systems) appear to have smaller grains than group II sources (selfshadowed-disk objects).

Two explanations are given here. Due to coagulation, dust particles grow. It is therefore possible that different grain sizes indicate different evolutionary stages. Group I sources have dust that is only marginally evolved compared to ISM dust. Group II sources on the other hand have a significant population of mm-sized grains. We suggest that flared disks, in which coagulation takes place, evolve into geometrically somewhat flatter self-shadowed disks (by dust settling to the midplane) and become group II members.

Alternatively, it is possible that, in contrast to coagulation, there are mechanisms that replenish the small-sized-grain population in circumstellar disks. The presence of the larger particles in self-shadowed-disk systems would then imply that this replenishment is not active/efficient (anymore) in these sources. In this hypothesis, flared disks are not necessarily evolutionally connected to self-shadowed disks, and the geometry of the disk is kept flared by the replenishment of the small grains.

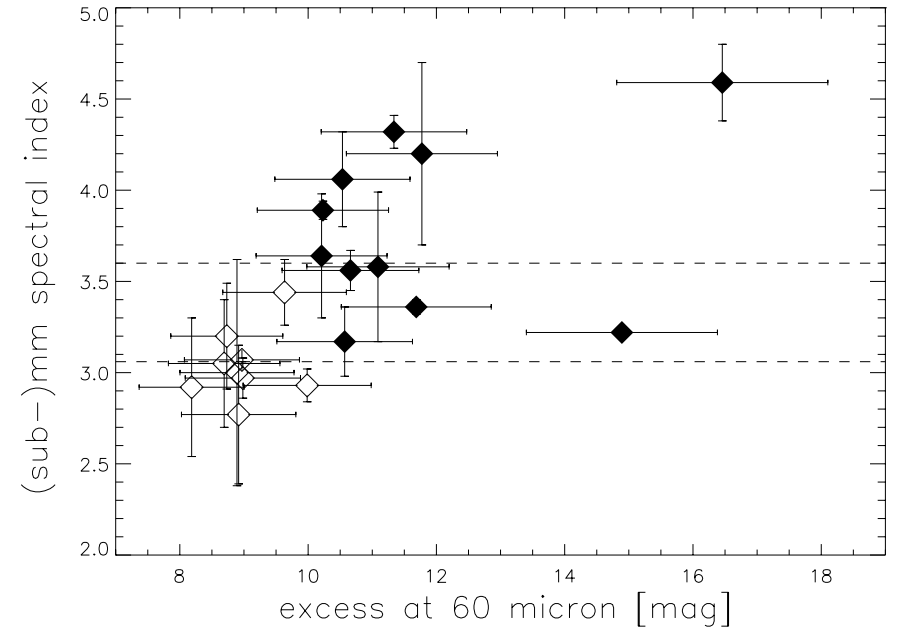

Fig. 3. The (sub-)mm spectral index versus the flux excess at $60 \mathrm{mi}-$ cron in magnitudes. Group I sources are represented by filled, group II by open symbols. The upper dashed line represents the average value of $n$ in group I, the lower line the average in group II.

We note that the shape of the amorphous 10 micron silicate feature, which is linked to the particle sizes of the warm silicates (Bouwman et al. 2001; van Boekel et al. 2003), does not give any direct information about the grain size distribution of the silicates in the mid-plane of the circumstellar disk. This feature is believed to originate from the surface layers of the disk. Grain growth of these warm grains has been observed in this sample of HAEBEs, but is uncorrelated with the (sub-)mm spectral index (see AV04). Hence these small $(\sim 0.1-2 \mu \mathrm{m})$ warm grains seem to have an evolution, independent of the grain growth in the interior of the disk. On the other hand, the 10 micron feature can be an indication for the sizes of the smallest grains in the disk, assuming that efficient vertical mixing in the disk occurs.

\section{Conclusions}

We showed in this paper that there is a significant difference between the (sub-)mm spectral indices of HAEBE stars with different IR SEDs. This could very well mean that the grain size distribution of the cold grains in a HAEBE star depends on the disk geometry.

The HAEBE systems with large IR excesses (group I, i.e. flaring-disk objects) have smaller cold grains than systems with more moderate IR excesses (group II, i.e. self-shadowed-disk sources). In other words, it appears that the flaring geometry of a circumstellar disk can only persist when a sufficiently large population of small grains exists in the disk.

The circumstellar-disk models of DD04 agree qualitatively with the results presented here. Disks in which the vast majority of the grains have grown to larger sizes, lead automatically to self-shadowed geometries. Models with small grains result in a mid-infrared SED akin to group I sources. Models with grain sizes larger than $\sim 1 \mathrm{~mm}$ not only display mid-infrared SEDs more resembling those of group II sources, but also change their (sub-)mm slope from larger values of $n$ to a black-body character $(n=3)$. DD04 note that the (sub-)mm slope changes 
with somewhat smaller grain sizes than are required to alter the mid-IR SED from group I to group II. Therefore, according to the models, the spectral index can have all values larger than 3 in group I sources, and all group II sources should have a black-body (sub-)mm slope. This is consistent with the observed trend in which group I sources are observed to have $3.17<n<4.59$ and group II sources have $n$ very close to 3. The DD04 models predict that grain growth resulting in a change of $n$ from values similar to those found in the ISM $(>3)$ to the optically thin black-body value $(n=3)$ occurs before the disk becomes self-shadowed. However the DD04 models do not model the dynamical processes leading to grain growth. At present the time scale of this transition from larger $n$ to $n=3$ is unknown, so we are unable to estimate the fraction of group I sources that should have $n=3$. Therefore we have no reason to assume a priori that our distribution of (sub-)mm-spectralindex values is incompatible with the interpretation outlined in Sect. 3.2.

In the present paper, we report a clear correlation between the shape of the mid-IR SED and the (sub-)mm slope in our sample. Previous authors have shown that variations in (sub-)mm slope within systems with proto-planetary disks are caused by variations in dust particle size. D02 have shown that the shape of the mid-IR SED is a proxy for disk geometry (flared versus self-shadowed). In accordance with these results, we interpret our find as a correlation between disk geometry and grain size. If one assumes that in the course of disk evolution grains will grow, this offers the first observational indications to the idea that flaring disks evolve into self-shadowed disks.

\section{References}

Acke, B., \& van den Ancker, M. E. 2004, A\&A, submitted Beckwith, S. V. W., \& Sargent, A. I. 1991, ApJ, 381, 250 Bouwman, J., de Koter, A., van den Ancker, M. E., \& Waters, L. B. F. M. 2000, A\&A, 360, 213

Bouwman, J., Meeus, G., de Koter, A., et al. 2001, A\&A, 375, 950

Chiang, E. I., \& Goldreich, P. 1997, ApJ, 490, 368

Colangeli, L., Battaglia, R., Brucato, J. R., et al. 2001, Meteoritics \& Planetary Science Suppl., 36, 44

Coulson, I. M., \& Walther, D. M. 1995, MNRAS, 274, 977

di Francesco, J., Evans, N. J., Harvey, P. M., et al. 1997, ApJ, 482, 433

Dullemond, C. P. 2002, A\&A, 395, 853

Dullemond, C. P., \& Dominik, C. 2004, A\&A, 417, 159

Dullemond, C. P., Dominik, C., \& Natta, A. 2001, ApJ, 560, 957
Dullemond, C. P., van den Ancker, M. E., Acke, B., \& van Boekel, R. 2003, ApJ, 594, L47

Fuente, A., Rodríguez-Franco, A., Testi, L., et al. 2003, ApJ, 598, L39

Gammie, C. F. 2001, ApJ, 553, 174

Henning, T., Burkert, A., Launhardt, R., Leinert, C., \& Stecklum, B. 1998, A\&A, 336, 565

Henning, T., Launhardt, R., Steinacker, J., \& Thamm, E. 1994, A\&A, 291, 546

Henning, T., Pfau, W., Zinnecker, H., \& Prusti, T. 1993, A\&A, 276, 129

Hillenbrand, L. A., Strom, S. E., Vrba, F. J., \& Keene, J. 1992, ApJ, 397,613

Jensen, E. L. N., Mathieu, R. D., \& Fuller, G. A. 1996, ApJ, 458, 312

Malfait, K., Bogaert, E., \& Waelkens, C. 1998, A\&A, 331, 211

Mannings, V. 1994, MNRAS, 271, 587

Mannings, V., \& Emerson, J. P. 1994, MNRAS, 267, 361

Mannings, V., \& Sargent, A. I. 1997, ApJ, 490, 792

Mannings, V., \& Sargent, A. I. 2000, ApJ, 529, 391

Meeus, G., Bouwman, J., Dominik, C., Waters, L. B. F. M., \& de Koter, A. 2003, A\&A, 402, 767

Meeus, G., Waters, L. B. F. M., Bouwman, J., et al. 2001, A\&A, 365, 476

Mennella, V., Brucato, J. R., Colangeli, L., et al. 1998, ApJ, 496, 1058

Miyake, K., \& Nakagawa, Y. 1993, Icarus, 106, 20

Natta, A., Grinin, V., \& Mannings, V. 2000, Protostars and Planets IV, 559

Natta, A., Grinin, V. P., Mannings, V., \& Ungerechts, H. 1997, ApJ, 491,885

Natta, A., Palla, F., Butner, H. M., Evans, N. J., \& Harvey, P. M. 1992, ApJ, 391, 805

Natta, A., Prusti, T., \& Krugel, E. 1993, A\&A, 275, 527

Natta, A., Prusti, T., Neri, R., et al. 1999, A\&A, 350, 541

Natta, A., Prusti, T., Neri, R., et al. 2001, A\&A, 371, 186

Natta, A., Testi, L., Neri, R., Shepherd, D., \& Wilner, D. 2004, A\&A, 416, 179

Osterloh, M., \& Beckwith, S. V. W. 1995, ApJ, 439, 288

Piétu, V., Dutrey, A., \& Kahane, C. 2003, A\&A, 398, 565

Sheret, I., Dent, W. R. F., \& Wyatt, M. C. 2004, MNRAS, 348, 1282

Sylvester, R. J., Skinner, C. J., Barlow, M. J., \& Mannings, V. 1996, MNRAS, 279, 915

Testi, L., Natta, A., Shepherd, D. S., \& Wilner, D. J. 2003, A\&A, 403, 323

Thé, P. S., de Winter, D., \& Pérez, M. R. 1994, A\&AS, 104, 315

Tuthill, P. G., Monnier, J. D., \& Danchi, W. C. 2001, Nature, 409, 1012

van Boekel, R., Waters, L. B. F. M., Dominik, C., et al. 2003, A\&A, 400, L21

van den Ancker, M. E., Bouwman, J., Wesselius, P. R., et al. 2000, A\&A, 357, 325

Walker, H. J., \& Butner, H. M. 1995, Astron. Space Sci., 224, 389 